\title{
DoUBLE JEOPARDY IN ACUTE ST-SEGMENT ELEVATION MYOCARDIAL INFARCTION
}

Singapore Med J 2017; 58(4): 225-227 doi: 10.11622/smedj.2017030

\section{Dear Sir,}

Acute multi-coronary thrombotic occlusion is an uncommon presentation of ST-segment elevation myocardial infarction (STEMI). Only a few reports of this condition have been published to date and the appropriate management options are unclear. ${ }^{(1)}$ The present report draws attention to simultaneous coronary thrombosis in the right coronary artery (RCA) and left anterior descending artery (LAD) and discusses the possible mechanism and treatment strategy.

A 51-year-old man presented with typical chest pain of four hours' duration and pulmonary oedema. His cardiovascular risk factors included Type 2 diabetes mellitus, hypertension, dyslipidaemia and smoking (ten cigarettes per day for the past 20 years). The patient received intranasal oxygen therapy, intravenous morphine and furosemide in the emergency department. A loading dose of aspirin $300 \mathrm{mg}$ and clopidogrel $600 \mathrm{mg}$ was administered. Electrocardiography revealed ST-segment elevation in the inferior leads. Coronary angiograms showed a thrombotic occlusion of the mid-RCA and the proximal LAD, with thrombolysis in myocardial infarction (TIMI) 0 flow (Figs. 1a \& b).

Primary percutaneous coronary intervention $(\mathrm{PCl})$ was performed via a 7 French $(\mathrm{Fr})$ sheath in the right common femoral artery. The patient received heparin anticoagulation during $\mathrm{PCl}$, guided by an activated clotting time. Initial results with plain balloon angioplasty were unsatisfactory for both the lesions, with a large thrombus burden. Thrombus was aspirated from the RCA and the LAD with a 7 Fr Thrombuster aspiration catheter (Kaneka Medix Corp, Shinagawa-ku, Tokyo, Japan). TIMI 3 flow was achieved in both the arteries, with a residual stenosis of $50 \%-60 \%$ in the mid-RCA and $60 \%-70 \%$ in the mid-LAD (Figs. 2a \& b). The coronary arteries were ectatic. An intra-aortic balloon pump was inserted for haemodynamic stability. During PCl, the patient had also received two boluses of eptifibatide $(180 \mu \mathrm{g} / \mathrm{kg}$ given ten minutes apart), followed by an infusion for 24 hours $(2.0 \mu \mathrm{g} / \mathrm{kg} / \mathrm{min})$. As reperfusion was successful following the aspiration thrombectomy, stenting during primary PCl was deferred. Following PCl, low-molecular-weight heparin ( $1 \mathrm{mg} / \mathrm{kg}$ body weight, twice daily) was administered during the period of index hospitalisation. Investigations did not reveal
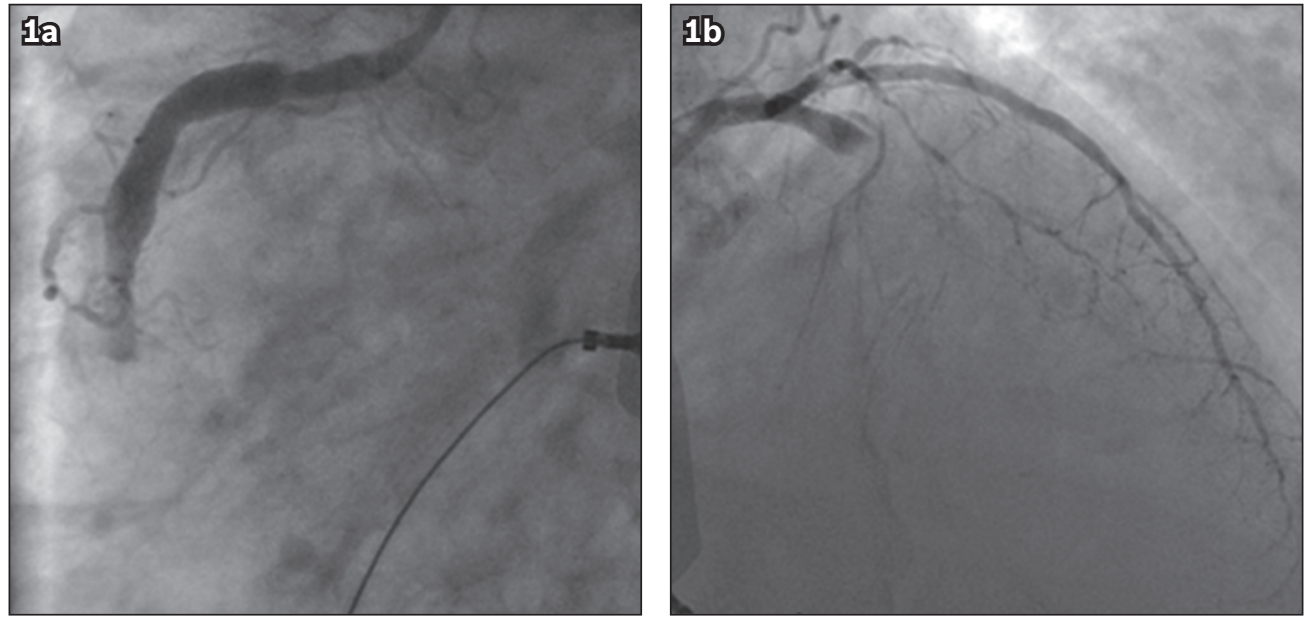

Fig. 1 Diagnostic coronary angiograms show an occluded (a) mid-right coronary artery and (b) proximal left anterior descending artery.
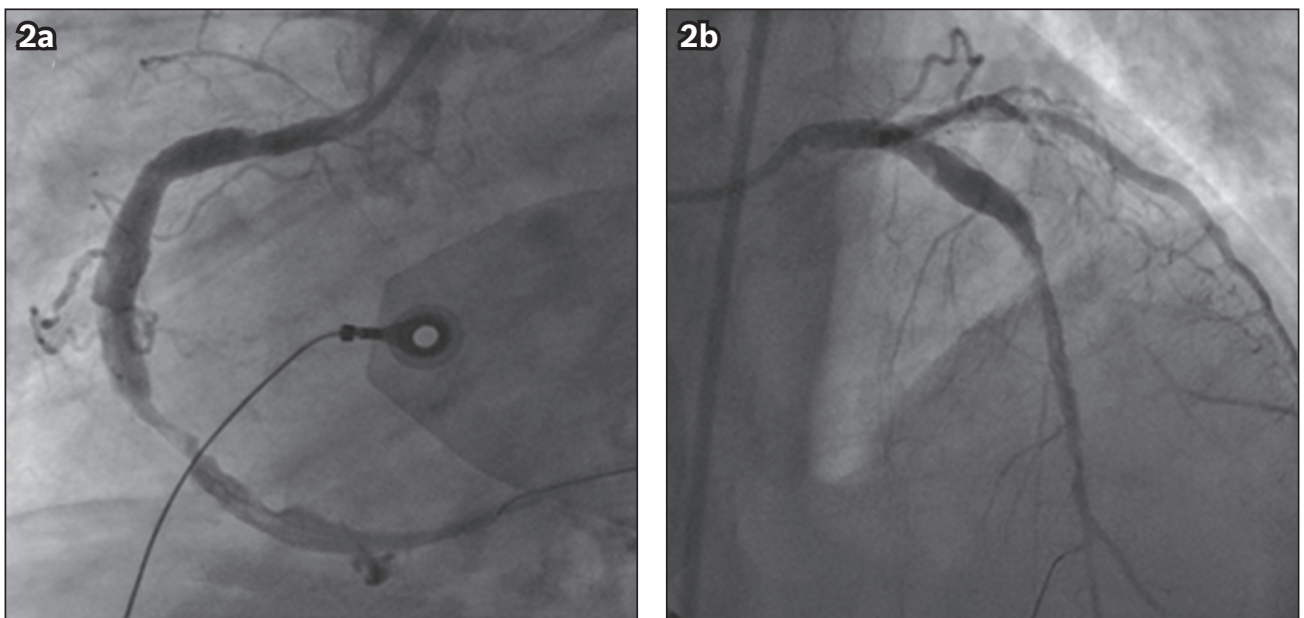

Fig. 2 Coronary angiograms show restoration of flow in the (a) right coronary artery, with a residual stenosis of $50 \%-60 \%$, and (b) left anterior descending artery, with a residual stenosis of $60 \%-70 \%$, following thrombus aspiration. The coronary arteries are ectatic. 

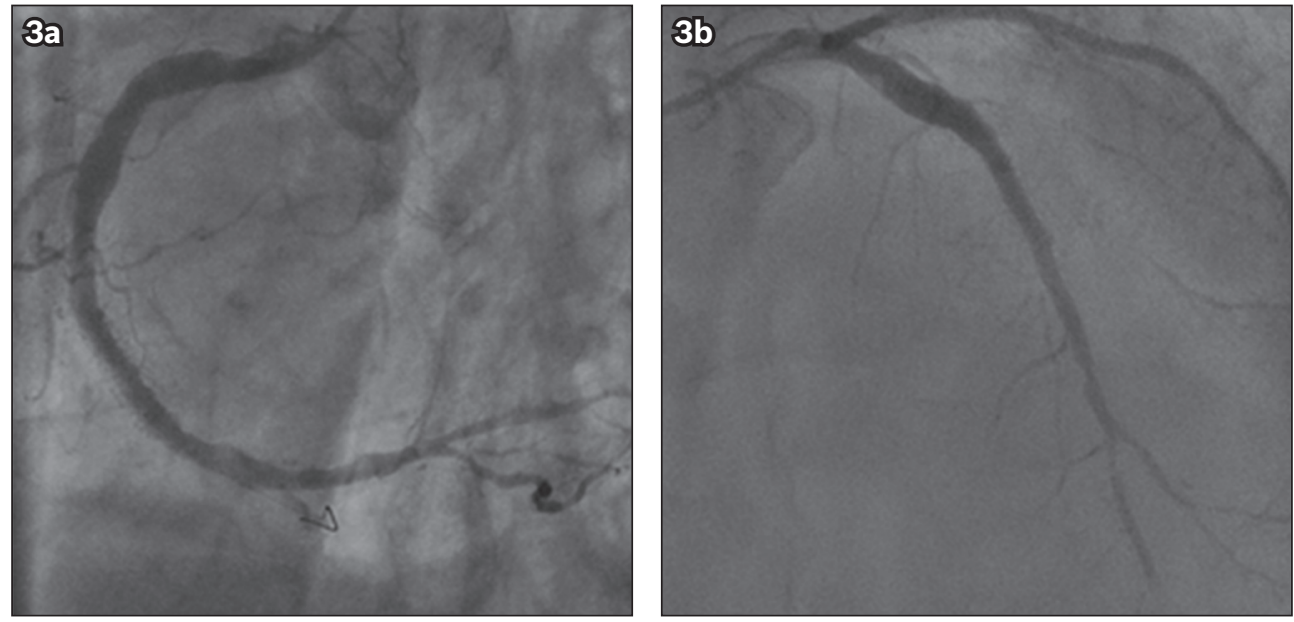

Fig. 3 Coronary angiograms show the outcome following the successful deployment of stents in the (a) right coronary artery and (b) left anterior descending artery.

an embolic source. Transthoracic echocardiography showed moderate left ventricular systolic dysfunction (ejection fraction 35\%), with regional wall motion abnormalities in RCA and LAD territory.

On Day 2, the patient was weaned off the intra-aortic balloon pump. There were no complications and the in-hospital course was uneventful. The patient was discharged on Day 5 with dual anti-platelet therapy (aspirin $100 \mathrm{mg}$ and clopidogrel $75 \mathrm{mg}$ once daily), a high-dose statin (simvastatin $40 \mathrm{mg}$ once daily), a beta-blocker (bisoprolol $2.5 \mathrm{mg}$ once daily), an angiotensin-converting enzyme inhibitor (lisinopril $2.5 \mathrm{mg}$ once daily), a loop diuretic (furosemide $40 \mathrm{mg}$ once daily) and an aldosterone antagonist (spironolactone $25 \mathrm{mg}$ once daily). One month later, the patient underwent a successful staged PCI involving stenting to the RCA and, subsequently, to the LAD (Figs. 3a \& b). A bare metal stent $(4.5 \mathrm{~mm} \times 20 \mathrm{~mm}$ OMEGA stent; Boston Scientific Corporation, Marlborough, MA, USA) was implanted in the mid-RCA lesion and a drug-eluting stent (COMBO $3.5 \mathrm{~mm} \times 15 \mathrm{~mm}$ stent; OrbusNeich Medical, Fort Lauderdale, FL, USA) was implanted in the mid-LAD lesion.

STEMI is caused by the rupture of an unstable plaque and commonly manifests as a single thrombotic occlusion on angiography. However, patients with STEMI have a diffuse inflammatory process, and vulnerable plaques in multiple coronary arteries are not uncommon. ${ }^{(2)}$ During primary $\mathrm{PCl}$, thrombosis affecting more than one coronary artery has been reported in $2.5 \%$ of cases. ${ }^{(3)}$ Identifiable causes include coronary vasospasm, cocaine abuse and hypercoagulable states. As our patient had none of the above, the most likely putative cause was simultaneous plaque ruptures leading to thrombotic occlusion in multiple coronary arteries.

A significant amount of myocardial jeopardy is expected in cases of acute thrombotic occlusion of more than one coronary tree. The majority of patients are haemodynamically unstable, and primary $\mathrm{PCl}$ is the reperfusion strategy of choice. Although routine aspiration thrombectomy during primary $\mathrm{PCl}$ is currently not recommended, it is a prudent choice in the presence of a large thrombus burden. Thrombus burden is an important risk factor for no reflow during primary PCl. No reflow occurs in $10 \%$ of cases of primary $\mathrm{PCl}$ and is associated with a larger infarct size, lower ejection fraction and increased risk of mortality. Independent predictors of its development after primary PCl are: a history of previous myocardial infarction, an elevated level of C-reactive protein at baseline, a large infarct size and lack of residual blood flow in the infarct-related artery. ${ }^{(4)}$ In our patient, the large size of the infarct and poor left ventricular systolic function were significant contributors to this phenomenon.

Management of acute multi-coronary thrombotic occlusions has been reported during the acute phase using multiple stents, or with only thrombus aspiration and haemodynamic support. ${ }^{(5,6)}$ It is important to emphasise that the primary goal of PCI in STEMI is rapid restoration of TIMI 3 flow in the culprit vessel. Stent implantation during primary PCI decreases the risk for subsequent target lesion and target vessel revascularisation, but is not associated with a reduction in the mortality rate..$^{(7)}$ In acute myocardial infarction, stenting can be avoided when successful reperfusion is established with thrombus aspiration alone, in the absence of a significant residual disease. ${ }^{(8)}$ An obvious concern is the risk of reocclusion in the presence of residual stenosis. Coronary stenting is most effective in preventing abrupt vessel closure from vascular recoil and vasospasm; the stent restores normal blood flow through its scaffolding effect. However, implantation of multiple coronary stents in the presence of a heavy thrombus burden and impaired blood flow increases the risk of procedural failure, due to the hazards of distal embolisation and stent thrombosis. The current medical therapy for STEMI is effective in plaque stabilisation, reduction of thrombus burden and prevention of reinfarction. Stent implantation can be scheduled at a later date after the patient's haemodynamic condition has stabilised and the plaques have been passivated, as in our case. This strategy was proven to be effective in our patient.

All the DEFER trials to date have discussed the outcome of culprit vessel revascularisation versus complete revascularisation, and deferred stent implantation versus conventional stent implantation during STEMI. ${ }^{(9-1)}$ Our patient had simultaneous thrombosis in LAD and RCA, which did not fulfil the criteria of the DEFER trials. Currently, there are no guidelines for optimal management strategy 
in patients with acute multi-vessel thrombotic occlusions. Most researchers agree that primary PCI for non-culprit lesions should be considered in patients with cardiogenic shock or persistent ischaemia.

To conclude, the possibility of multi-vessel coronary thrombosis should be considered during primary $\mathrm{PCl}$, especially in an unstable patient. Expeditious restoration of coronary blood flow will salvage the myocardium and reduce mortality risk. As shown in our patient, stenting can be staged in the absence of critical flow-limiting disease, if successful reperfusion is established with balloon dilatation and thrombus aspiration.

Yours sincerely,

Rajiv Ananthakrishna ${ }^{1}$, Li-Jun $\underline{\text { Wang}}{ }^{1}$, Liang Ping $\underline{Z h a o}^{1}$, Huay Cheem $\underline{\text { Tan }}^{1}$

${ }^{1}$ Department of Cardiology, National University Heart Centre, Singapore. rajiva.ms@gmail.com

\section{References}

1. Mahmoud A, Saad M, Elgendy IY. Simultaneous multi-vessel coronary thrombosis in patients with ST-elevation myocardial infarction: a systematic review. Cardiovasc Revasc Med 2015; 16:163-6.

2. Asakura M, Ueda $\mathrm{Y}$, Yamaguchi O, et al. Extensive development of vulnerable plaques as a pan-coronary process in patients with myocardial infarction: an angioscopic study. J Am Coll Cardiol 2001; 37:1284-8.

3. Pollak PM, Parikh SV, Kizilgul M, Keeley EC. Multiple culprit arteries in patients with ST segment elevation myocardial infarction referred for primary percutaneous coronary intervention. Am J Cardiol 2009; 104:619-23.

4. Ndrepepa G, Tiroch K, Keta D, et al. Predictive factors and impact of no reflow after primary percutaneous coronary intervention in patients with acute myocardial infarction. Circ Cardiovasc Interv 2010; 3:27-33.

5. Nazer B, Hayward RM, Boyle AJ. Simultaneous thrombotic culprit lesions in two separate coronary arteries in a patient with ST-elevation myocardial infarction. Eur Heart J 2012; 33:2622.

6. Turgeman Y, Suleiman K, Atar S. Multivessel acute coronary thrombosis and occlusion--an unusual cause of cardiogenic shock. J Invasive Cardiol 2007; 19:E278-80.

7. Nordmann AJ, Hengstler P, Harr T, Young J, Bucher HC. Clinical outcomes of primary stenting versus balloon angioplasty in patients with myocardial infarction: a meta-analysis of randomized controlled trials. Am J Med 2004; 116:253-62.

8. Escaned J, Echavarría-Pinto M, Gorgadze T, et al. Safety of lone thrombus aspiration without concomitant coronary stenting in selected patients with acute myocardial infarction. Eurolntervention 2013; 8:1149-56.

9. Vlaar PJ, Mahmoud KD, Holmes DR Jr, et al. Culprit vessel only versus multivessel and staged percutaneous coronary intervention for multivessel disease in patients presenting with ST-segment elevation myocardial infarction: a pairwise and network meta-analysis. J Am Coll Cardiol 2011; 58:692-703.

10. Engstrøm T, Kelbæk H, Helqvist S, et al. Complete revascularisation versus treatment of the culprit lesion only in patients with ST-segment elevation myocardial infarction and multivessel disease (DANAMI-3_PRIMULTI): an open-label, randomised controlled trial. Lancet 2015; 386:665-71.

11. Kelbæk H, Høfsten DE, Køber L, et al. Deferred versus conventional stent implantation in patients with ST-segment elevation myocardial infarction (DANAMI 3-DEFER): an open-label randomised controlled trial. Lancet 2016; 387:2199-206. 